\title{
Effects of Extracorporeal Shockwave Therapy in Chronic Stroke Patients With Knee Osteoarthritis: A Pilot Study
}

\author{
Sung Jun Cho, MD, Ja Ryung Yang, MD, Hee Seung Yang, MD, Hea-Eun Yang, MD
}

Department of Physical Medicine and Rehabilitation, Veterans Health Service Medical Center, Seoul, Korea

\begin{abstract}
Objective To evaluate the effects of extracorporeal shockwave therapy (ESWT) on pain, function, and ultrasonographic features of chronic stroke patients with knee osteoarthritis (OA).

Methods A total of 18 chronic stroke patients (33 knee joints) with unilateral or bilateral knee OA (KellgrenLawrence grade $\geq 1$ ) were enrolled in this study. The patients were randomly allocated to an experimental group receiving ESWT $(n=9)$ or a control group receiving sham ESWT $(n=9)$. For the ESWT group, patients received 1,000 pulses weekly for 3 weeks, totaling to an energy dose of $0.05 \mathrm{~mJ} / \mathrm{mm}^{2}$ on the proximal medial tibia of the affected knee. The assessments were performed before the treatment, immediately after the first treatment, and 1 week after the last treatment using the following: the visual analog scale (VAS) for pain; patient perception of the clinical severity of OA; the Korean version of Modified Barthel Index (ambulation and chair/bed transfer); the Functional Independence Measure scale (FIM; bed/chair/wheelchair transfer, toilet transfer, walking, and stairs); and ultrasonographic features (articular cartilage thickness, Doppler activity, and joint effusion height).

Results The experimental group showed a significant improvement in VAS score $(4.50 \pm 1.87$ to $2.71 \pm 1.38)$ and patient perception of the clinical severity of OA $(1.87 \pm 0.83$ to $2.75 \pm 0.46)$. The bed/chair/wheelchair transfer components of the FIM score also improved significantly (4.12 \pm 1.55 to $4.62 \pm 1.30$ ). In terms of the ultrasonographic features, increased Doppler activity was observed in the medial knee in the experimental group immediately following ESWT.

Conclusion It is suggested that ESWT may reduce pain and improve function in chronic stroke patients with OA, and may increase vascular activity at the target site.
\end{abstract}

Keywords High-energy shock waves, Knee osteoarthritis, Stroke, Ultrasonography, Pain

Received December 9, 2015; Accepted February 2, 2016

Corresponding author: Hea-Eun Yang

Department of Physical Medicine and Rehabilitation, Veterans Health Service Medical Center, 53 Jinhwangdo-ro 61-gil, Gangdong-gu, Seoul 05368, Korea. Tel: +82-2-2225-1303, Fax: +82-2-2225-1579, E-mail: yangmary429@gmail.com

ORCID: Sung Jun Cho (http://orcid.org/0000-0002-0297-0049); Ja Ryung Yang (http://orcid.org/0000-0003-0581-5041); Hee Seung Yang (http://orcid. org/0000-0003-2492-7065); Hea-Eun Yang (http://orcid.org/0000-0002-4449-7288).

(a) This is an open-access article distributed under the terms of the Creative Commons Attribution Non-Commercial License (http://creativecommons.org/ licenses/by-nc/4.0) which permits unrestricted noncommercial use, distribution, and reproduction in any medium, provided the original work is properly cited. Copyright $\odot 2016$ by Korean Academy of Rehabilitation Medicine 


\section{INTRODUCTION}

Knee osteoarthritis (OA) is a common musculoskeletal disease and is a leading cause of pain and disability worldwide [1]. Knee OA negatively affects physical function and quality of life. The prevalence of radiographic knee OA and symptomatic knee OA in Korean elderly community residents (age $>50$ years) is $37.3 \%$ and $24.2 \%$, respectively [2].

In stroke patients, OA can be a comorbid pathology that has a significant impact on the stroke outcome, particularly considering its increasing prevalence with age [3]. Knee OA can be accelerated due to an alteration of normal biomechanics in stroke patients. Knee OA has a negative effect on ambulation levels in post-stroke hemiplegic patients, and it must therefore be taken into consideration during the planning of a stroke rehabilitation program [3].

Optimal management of patients with knee OA requires a combination of non-pharmacological and pharmacological modalities, and surgical treatment is recommended in intractable cases [4]. Non-pharmacological modalities include education, muscle strengthening, thermal modalities, and transcutaneous electrical nerve stimulation, among others, while pharmacological treatment modalities range from oral medication to intraarticular injections.

In recent decades, extracorporeal shockwave therapy (ESWT) has been widely used for pain relief and treatment of musculoskeletal disorders [5]. The effects of ESWT in patients with plantar fasciitis, calcifying shoulder tendinitis, and lateral epicondylitis of the elbow have been reported, and clinical applications of ESWT in patients with these diseases are widely used [5].

Veterinarians have started using ESWT to treat equine knee OA $[6,7]$. It has been reported that the application of ESWT on arthritic joints in animals improves limb motor dysfunction $[8,9]$. Recent studies reported that, histopathologically, ESWT has a chondroprotective effect in small animals, such as rats and rabbits [10-12]. Since ESWT is non-invasive, has a low complication rate, does not require hospitalization, and is relatively inexpensive compared to other conservative and surgical approaches, it is the preferred treatment option for knee OA. To our knowledge, only a few studies have investigated its use in human knee OA, and these studies have assessed only the clinical outcomes and not the morphological or structural changes in the knee joint.

This study was a pilot study that investigated the effects of ESWT in chronic stroke patients with knee OA in terms of pain, function, and morphological changes assessed with ultrasonography.

\section{MATERIALS AND METHODS}

\section{Participants}

The present study was a prospective, double-blind, randomized controlled trial. Patients were recruited from an inpatient rehabilitation center. The inclusion criteria were as follows: chronic stroke of $\geq 2$ years duration; unilateral or bilateral knee OA (Kellgren-Lawrence grade $\geq 1$ ); intact cognition (Mini-Mental State Examination score $>20$ ); and ambulatory ability. Patients with other musculoskeletal conditions that can cause lower extremity pain, secondary causes of arthritis, or history of intraarticular knee injection within the previous 6 months were excluded.

A total of 18 chronic stroke patients with knee OA were recruited, and a total of 33 knees were analyzed in the present study. The study protocol was approved by the Institutional Review Board.

\section{Intervention}

For this randomized controlled study, a randomization sequence was generated by a computer program prior to the start of the study. This procedure was performed by an independent researcher who was not involved in the intervention or assessments. The patients were allocated to 1 of the following: the experimental group $(n=9,16$ knee joints) receiving ESWT or the control group ( $n=9,17$ knee joints) receiving sham ESWT. Patients were not informed of the group assignment. The patients were then referred to other 2 researchers assessing pain, functional scales and ultrasonographic features of subjects, respectively.

ESWT and sham ESWT were administered by a physiatrist (SJ Cho) majoring in rehabilitation medicine who have performed ESWT treatments for more than 3 years and was not involved in the randomization or assessments. Shockwaves were delivered using Dornier Aries (Dornier MedTech, Wessling, Germany); patients underwent 3 sessions at weekly intervals. For the session, pa- 
tients were in the supine position with the affected knee flexed and the foot on the table. At each treatment session, the experimental group received 1,000 impulses of shockwave at $0.05 \mathrm{~mJ} / \mathrm{mm}^{2}$ on the proximal medial tibia of the affected knee. The control group received 1,000 impulses of sham stimulation at $0 \mathrm{~mJ} / \mathrm{mm}^{2}$ on the same area.

\section{Outcomes}

Overall, 3 domains were assessed; pain, functional state, and ultrasonographic features. Evaluations were performed at baseline and 1 week after the final treatment session for all participants, but the Doppler activity on the ultrasonography was performed immediately after the first treatment session. All outcome measurements were assessed by 2 researchers who were not involved in the randomization or intervention.

For the pain evaluation, the visual analog scale (VAS) score [13] and patient perception of the clinical severity of OA (PPOA) [14] were assessed. PPOA is a 5-point scoring system that reflects patient consideration about the effect of knee OA on their daily lives, with 1 indicating very poor and 5 indicating very good.

To assess the functional state, specific domains involving the lower extremity in the Korean version of the Modified Barthel Index (K-MBI) [15] and Functional Independence Measure (FIM) scale [16] were used. Ambulation and chair/bed transfer ability from the K-MBI and bed/ chair/wheelchair transfer, toilet transfer, walking, and stairs ability from the FIM scale were extracted.

For the ultrasonographic features, we extracted meaningful domains from the standardized musculoskeletal ultrasound (MUS) scoring system for knee OA [17]. Articular cartilage thickness was measured at the medial trochlea, trochlear notch, and lateral trochlea. Joint effusion height was measured at the suprapatellar recess. Doppler activity was assessed at the medial joint space, medial parapatellar recess, lateral joint space, and lateral parapatellar recess. Articular cartilage thickness was measured in every participant, and the Doppler activity and joint effusion were assessed in 5 participants (8 knee joints) from the experimental group and 4 participants ( 7 knee joints) from the control group.

\section{Statistical analysis}

All analyses were performed using Statistical Package for Social Sciences (SPSS) software ver. 18.0 (SPSS Inc., Chicago, IL, USA). A paired t-test was used to compare within-group changes in the measured parameters, and an independent t-test was conducted to determine statistical differences in the variables between the 2 groups. The statistical differences of each segment in terms of the Doppler activity data were analyzed using repeated measures ANOVA. Values are presented as mean \pm standard deviation, and $\mathrm{p}<0.05$ was considered statistically significant.

\section{RESULTS}

All 18 patients completed the treatment sessions without any complications. There were no significant differences between the experimental and control groups in terms of the baseline characteristics and parameters (Table 1).

The experimental group showed reduced pain, as measured on the VAS and PPOA scales after the intervention (Table 2). The VAS score was $4.5 \pm 1.9$ at baseline and 2.7 \pm 1.4 after the treatment $(\mathrm{p}=0.00)$. The PPOA

Table 1. Demographic and clinical characteristics

\begin{tabular}{lccc}
\hline & Experimental group & Control group & p-value \\
\hline Age (yr) & $75.5 \pm 7.7$ & $72.7 \pm 5.9$ & 0.43 \\
Body weight $(\mathrm{kg})$ & $63.1 \pm 9.8$ & $61.0 \pm 10.7$ & 0.69 \\
Height $(\mathrm{cm})$ & $166.0 \pm 5.8$ & $161.2 \pm 9.1$ & 0.23 \\
Sex (female:male) & $1: 8$ & $2: 7$ & 0.13 \\
\hline MMSE & $21.1 \pm 5.4$ & $22.8 \pm 4.7$ & 0.53 \\
\hline K-L grade & $2.0 \pm 1.1$ & $1.8 \pm 1.1$ & 0.75 \\
\hline
\end{tabular}

Values are presented as mean \pm standard deviation or number of subjects.

MMSE, Mini-Mental State Examination; K-L grade, Kellgren-Lawrence grade. 
Table 2. Pre- and post-treatment pain scores

\begin{tabular}{|lccc}
\hline & Baseline & 1 week after the final treatment & p-value $^{\text {a) }}$ \\
\hline VAS & & & $0.00^{*}$ \\
\hline Experimental group & $4.5 \pm 1.9$ & $2.7 \pm 1.4$ & 0.08 \\
\hline Control group & $4.3 \pm 1.9$ & $4.1 \pm 1.7$ & $0.02^{*}$ \\
\hline PPA & & & 0.60 \\
\hline Experimental group & $1.9 \pm 0.8$ & $2.8 \pm 0.5$ & $1.6 \pm 0.7$ \\
\hline Control group & $1.5 \pm 0.5$ & & 0 \\
\hline
\end{tabular}

Values are presented as mean \pm standard deviation.

VAS, visual analog scale; PPOA, patient perception of the clinical severity of osteoarthritis.

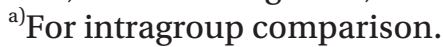

${ }^{*} \mathrm{p}<0.05$.

Table 3. Pre- and post-treatment functional domain scores

\begin{tabular}{|c|c|c|c|}
\hline & Baseline & 1 week after the final treatment & p-value ${ }^{a)}$ \\
\hline \multicolumn{4}{|l|}{ K-MBI (ambulation) } \\
\hline Experimental group & $9.3 \pm 3.7$ & $9.9 \pm 2.7$ & 0.35 \\
\hline Control group & $8.1 \pm 4.1$ & $8.4 \pm 3.7$ & 0.35 \\
\hline \multicolumn{4}{|c|}{ K-MBI (chair/bed transfer) } \\
\hline Experimental group & $9.3 \pm 3.7$ & $9.9 \pm 2.7$ & 0.35 \\
\hline Control group & $7.5 \pm 4.4$ & $7.8 \pm 4.2$ & 0.35 \\
\hline \multicolumn{4}{|l|}{ FIM (walking) } \\
\hline Experimental group & $4.6 \pm 1.8$ & $4.9 \pm 1.6$ & 0.17 \\
\hline Control group & $4.0 \pm 2.0$ & $4.3 \pm 1.8$ & 0.17 \\
\hline \multicolumn{4}{|l|}{ FIM (stairs) } \\
\hline Experimental group & $3.0 \pm 2.3$ & $3.1 \pm 2.2$ & 0.35 \\
\hline Control group & $2.5 \pm 1.8$ & $2.6 \pm 1.8$ & 0.35 \\
\hline \multicolumn{4}{|c|}{ FIM (bed/chair/wheelchair transfer) } \\
\hline Experimental group & $4.1 \pm 1.6$ & $4.6 \pm 1.3$ & $0.03^{*}$ \\
\hline Control group & $3.6 \pm 2.1$ & $4.0 \pm 1.8$ & 0.08 \\
\hline \multicolumn{4}{|l|}{ FIM (toilet transfer) } \\
\hline Experimental group & $4.0 \pm 1.6$ & $4.4 \pm 1.5$ & 0.08 \\
\hline Control group & $3.6 \pm 2.1$ & $3.8 \pm 1.9$ & 0.35 \\
\hline
\end{tabular}

Values are presented as mean \pm standard deviation.

K-MBI, Korean version of Modified Barthel Index; FIM, Functional Independence Measure.

${ }^{\text {a) }}$ For intragroup comparison.

${ }^{*} \mathrm{p}<0.05$.

score was $1.9 \pm 0.8$ at baseline and $2.8 \pm 0.5$ after the treatment ( $\mathrm{p}=0.02$ ). The control group showed no significant change in the pain domain. In the functional domain, the control group showed no significant change after all the treatment sessions. The experimental group showed an improvement in the bed/chair/wheelchair transfer component of the FIM score, but not in other components of the FIM or K-MBI (Table 3). The bed/chair/wheelchair transfer score on the FIM scale was $4.1 \pm 1.6$ at baseline and $4.6 \pm 1.3$ after the intervention in the experimental group $(\mathrm{p}=0.03)$.

The control group showed no significant change after the intervention in any of the domains of ultrasonographic features. The Doppler activity was evaluated using the following scoring system $(0$, none; 1 , less than half of the area of interest; 2 , more than half of the area of interest) 
[17]. An increase in Doppler activity was observed in the medial joint in the experimental group immediately after the first ESWT session. The Doppler activity score of the medial joint was $0.0 \pm 0.0$ at baseline and $0.8 \pm 0.7$ immediately after the ESWT $(\mathrm{p}=0.04)$. However, a decreased tendency was observed in the Doppler activity at 1 week af- ter all the treatment sessions $(0.5 \pm 0.5)$, compared to that obtained immediately after the ESWT $(0.8 \pm 0.7)$, although it was not statistically significant $(\mathrm{p}=0.08)$. No change was noted in other ultrasonographic findings, such as articular cartilage thickness, joint effusion, and Doppler activity in other parts excluding the medial knee joint (Tables 4, 5).

Table 4. Pre- and post-treatment articular cartilage thickness and joint effusion height

\begin{tabular}{|c|c|c|c|}
\hline & Baseline & 1 week after the final treatment & p-value ${ }^{\text {a) }}$ \\
\hline \multicolumn{4}{|c|}{ Articular cartilage thickness } \\
\hline \multicolumn{4}{|l|}{ Medial } \\
\hline Experimental group & $2.2 \pm 0.5$ & $2.2 \pm 0.5$ & 0.67 \\
\hline Control group & $1.9 \pm 0.5$ & $2.0 \pm 0.5$ & 0.12 \\
\hline \multicolumn{4}{|l|}{ Notch } \\
\hline Experimental group & $2.4 \pm 0.6$ & $2.2 \pm 0.4$ & 0.07 \\
\hline Control group & $2.1 \pm 0.6$ & $2.2 \pm 0.6$ & 0.24 \\
\hline \multicolumn{4}{|l|}{ Lateral } \\
\hline Experimental group & $2.1 \pm 0.4$ & $2.2 \pm 0.4$ & 0.14 \\
\hline Control group & $2.0 \pm 0.5$ & $2.1 \pm 0.5$ & 0.12 \\
\hline \multicolumn{4}{|l|}{ Joint effusion height } \\
\hline \multicolumn{4}{|c|}{ Suprapatellar recess space } \\
\hline Experimental group & $1.5 \pm 2.7$ & $1.4 \pm 2.6$ & 0.27 \\
\hline Control group & $2.2 \pm 2.8$ & $2.2 \pm 2.7$ & 0.90 \\
\hline
\end{tabular}

Values are presented as mean \pm standard deviation.

${ }^{a)}$ For intragroup comparison.

Table 5. Pre- and post-treatment Doppler activity

\begin{tabular}{|c|c|c|c|c|}
\hline & Baseline & $\begin{array}{l}\text { Immediately after } \\
\text { the first treatment }\end{array}$ & $\begin{array}{c}1 \text { week after } \\
\text { the final treatment }\end{array}$ & p-value ${ }^{\text {a) }}$ \\
\hline \multicolumn{5}{|l|}{ Doppler activity } \\
\hline \multicolumn{5}{|l|}{ Medial joint } \\
\hline Experimental group & $0.0 \pm 0.0$ & $0.8 \pm 0.7^{*}$ & $0.5 \pm 0.5$ & $0.04^{*}$ \\
\hline Control group & $0.3 \pm 0.5$ & $0.3 \pm 0.5$ & $0.3 \pm 0.5$ & 1.00 \\
\hline \multicolumn{5}{|c|}{ Medial parapatellar recess } \\
\hline Experimental group & $0.1 \pm 0.4$ & $0.1 \pm 0.4$ & $0.1 \pm 0.4$ & 1.00 \\
\hline Control group & $0.3 \pm 0.5$ & $0.3 \pm 0.5$ & $0.3 \pm 0.5$ & 1.00 \\
\hline \multicolumn{5}{|l|}{ Lateral joint } \\
\hline Experimental group & $0.0 \pm 0.0$ & $0.0 \pm 0.0$ & $0.0 \pm 0.0$ & 1.00 \\
\hline Control group & $0.1 \pm 0.4$ & $0.1 \pm 0.4$ & $0.1 \pm 0.4$ & 1.00 \\
\hline \multicolumn{5}{|c|}{ Lateral parapatellar recess } \\
\hline Experimental group & $0.0 \pm 0.0$ & $0.0 \pm 0.0$ & $0.0 \pm 0.0$ & 1.00 \\
\hline Control group & $1.0 \pm 0.8$ & $1.0 \pm 0.8$ & $1.0 \pm 0.8$ & 1.00 \\
\hline
\end{tabular}

Values are presented as mean \pm standard deviation.

${ }^{a)}$ For intragroup comparison between baseline and immediately after the first treatment. ${ }^{*} \mathrm{p}<0.05$. 
Increased Doppler activity was observed in diverse parts of the medial knee joint in each patient and most commonly on the distal femoral and proximal tibial surfaces (Fig. 1). In some patients, Doppler activity was also increased on the medial ligament or medial meniscus (Fig. 2).

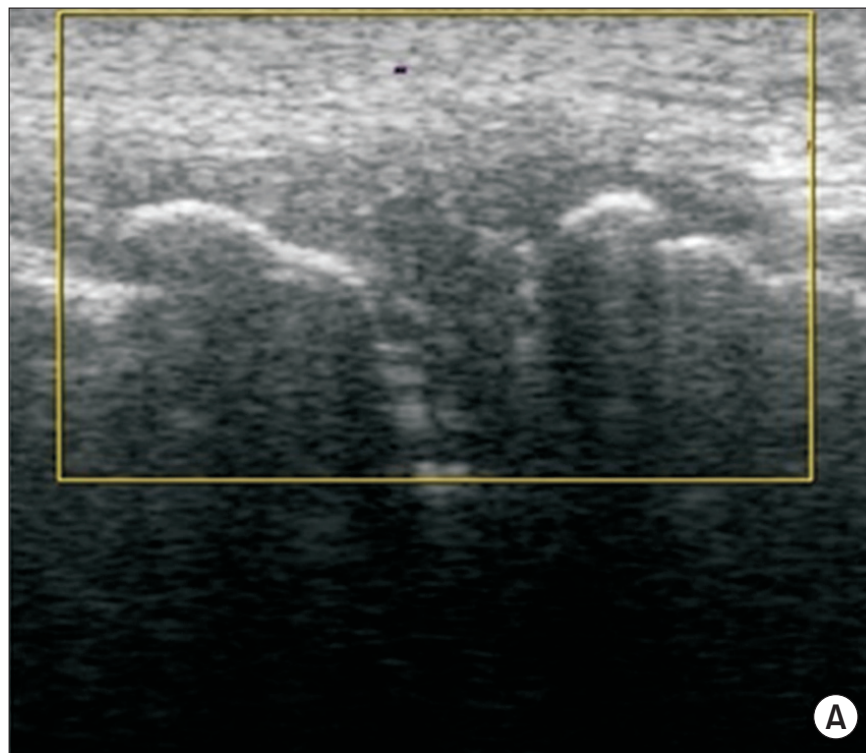

\section{DISCUSSION}

The present pilot study explored the effects of ESWT in chronic stroke patients with knee OA in terms of pain, function, and ultrasonographic features. Participant recruitment and treatment adherence were considered successful, and there were no adverse events related to the intervention. It is proposed that ESWT may increase

Fig. 1. Doppler activity from ultrasonography in the medial joint in the experimental group: (A) before and (B) immediately after the first ESWT session. Increased Doppler activity was observed in the medial joint and most commonly on the distal femoral and proximal tibial surfaces. ESWT, extracorporeal shockwave therapy.
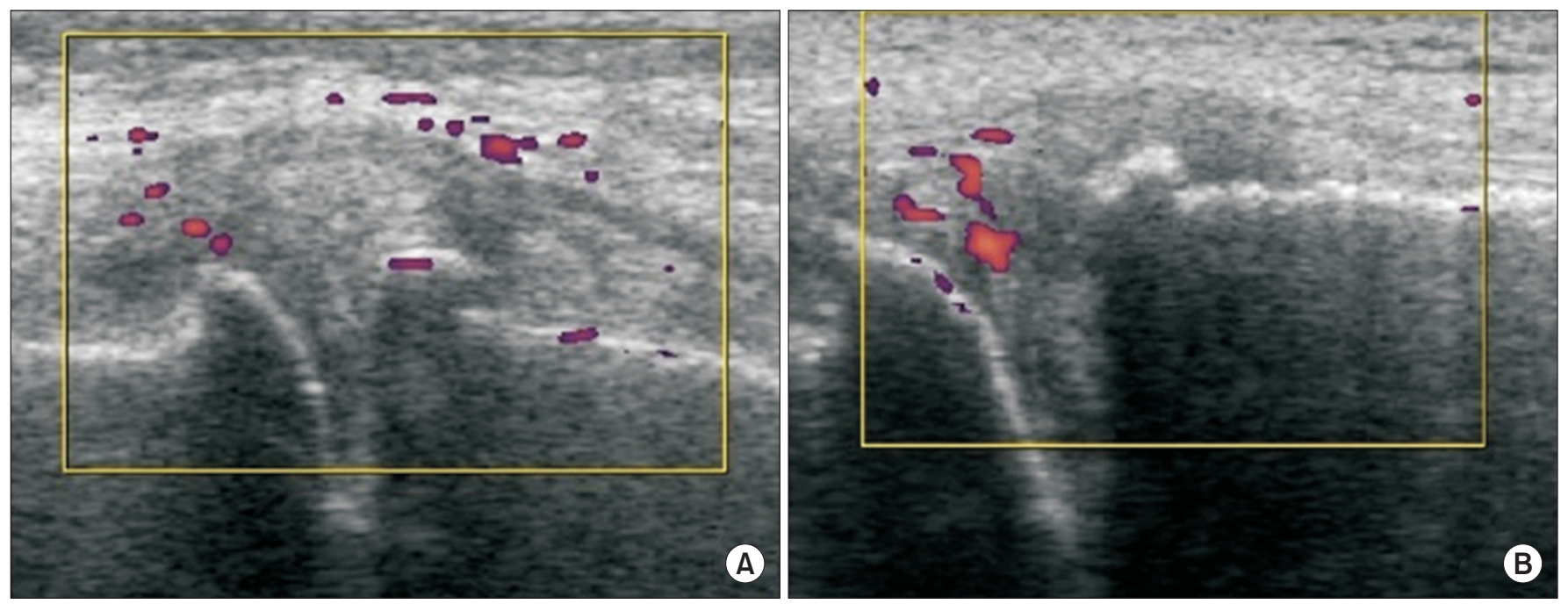

Fig. 2. Doppler activity from ultrasonography of the medial joint in the experimental group. Doppler activity was also increased on the medial ligament (A) or the medial meniscus (B). 
site-specific Doppler activity, reduce pain and improve motor function.

ESWT has been applied in various musculoskeletal disorders, including calcifying shoulder tendonitis, lateral epicondylitis of the elbow, and proximal plantar fasciitis. Recently, ESWT has been proposed as a therapeutic modality for knee OA. The exact mechanism underlying ESWT is unknown, but several studies suggest that shockwaves stimulate the early expression of angiogenesis-related growth factors, and then induce the ingrowth of neovascularization that improves blood supply and increases cell proliferation and eventual tissue regeneration to repair tendon or bone tissues $[5,18]$.

The chondroprotective effect of ESWT in OA has been established in animal studies. Wang et al. [10,11,19] performed a histomorphological examination and immunochemical analysis after the application of ESWT in the osteoarthritic knee in rats. Their studies demonstrated that ESWT protects articular cartilage degradation and improves subchondral bone remodeling in rats. The changes in the Mankin score, Safranin O staining, matrix metalloproteinase 13 (MMP-13), and collagen II were significant indicators of the influence of EWST on articular cartilage. The effects of ESWT in subchondral bone remodeling were supported by the increased vascularization manifested by the changes in the von Willebrand factor (vWF), endothelial nitric oxide synthase (eNOS), vessel endothelial growth factor (VEGF), and by the osteogenesis manifested by the changes in bone morphogenetic protein 2 (BMP-2), alkaline phosphatase, and osteocalcin. Zhao et al. [12] demonstrated that ESWT significantly reduces the nitric oxide level in the synovial cavity of knee joints and chondrocyte apoptosis in rabbits with $\mathrm{OA}$, thereby reducing the progression of OA.

In spite of this, its effects on human knee OA have not been studied sufficiently. There are only 2 previous human studies, and these studies proved that ESWT is effective in reducing pain and improving knee function. However, only the clinical outcomes related to knee OA were assessed, and the substantial morphological or hemodynamic changes in the knee joints were not included $[20,21]$.

Prior to the application of ESWT, the target area and dosage had to be determined. There is no stated target site for ESWT in knee OA. In humans, ESWT was applied on a trigger point around the knee or on the patellofemo- ral and tibiofemoral borders of the knee [20]. In rabbits, ESWT was applied around the knee [12]. In rats, ESWT was applied on the distal femur or proximal tibia, and a chondroprotective effect was seen at either site [11]. As different target sites were chosen in each study, we could not have solely referred to a single study in selecting a target site. Therefore, we have targeted at the subchondral bone of the medial proximal tibia that were commonly conducted in the studies. And we performed ESWT treatments on the same area for all patients for the unity of the intervention method. Also, there were some patients who had no tender point around the knee. For that reason, the tender point was not chosen as a target site. Additionally, in this study, veins, nerves, and ligaments were checked using ultrasound, and ESWT was administered at the target site, avoiding those structures.

Safety was the first priority when determining the appropriate dose. A single bout of ESWT (150 shockwaves of $0.5 \mathrm{~mJ} / \mathrm{mm}^{2}$ ) caused degenerative changes in the hyaline cartilage of an adult rat, on molecular and ultrastructural levels [22]. A study on the effects of ESWT on human chondrocytes in vitro revealed a dose-dependent increase in cytotoxicity [23]. Cytotoxicity was below $10 \%$ when using energy densities of $0.02 \mathrm{~mJ} / \mathrm{mm}^{2}$ and $0.06 \mathrm{~mJ} / \mathrm{mm}^{2}$, but the cytotoxicity increased up to approximately $20 \%$ when using $0.17 \mathrm{~mJ} / \mathrm{mm}^{2}$. A recently published study reported that medium energy $(0.093 \mathrm{~mJ} /$ $\mathrm{mm}^{2}$ ) ESWT was more effective in relieving pain and improving functional outcomes than low energy $(0.040 \mathrm{~mJ} /$ $\mathrm{mm}^{2}$ ) ESWT. However, histological or molecular safety evaluations were not carried out in that study [21].

According to a study conducted on human chondrocytes in vitro [23], cytotoxicity was measured below $10 \%$ with the dose of less than $0.06 \mathrm{~mJ} / \mathrm{mm}^{2}$. From the study, we have concluded that a dose of less than $0.06 \mathrm{~mJ} / \mathrm{mm}^{2}$ was an appropriate level for safety. In addition, a human study concerning the effect of different levels of energy revealed that ESWT energy above $0.04 \mathrm{~mJ} / \mathrm{mm}^{2}$ was more effective [21]. In consideration of these 2 studies, we applied an ESWT dose of $0.05 \mathrm{~mJ} / \mathrm{mm}^{2}$ in our study. Also, the latter human study [21] indicated that 1,000 shockwaves of ESWT had no side effects or complications, and so we applied the same times of shockwaves in our study.

In our study, an energy density of $0.05 \mathrm{~mJ} / \mathrm{mm}^{2}$ was selected based on a previous study of human chondrocytes, even though it was an in vitro study. 
In chronic stoke patients, it is difficult to expect functional recovery, especially if a specific comorbidity, such as knee OA, exists [3]. In this study, the bed/chair/wheelchair transfer component of the FIM scale was improved in the experimental group. This included all aspects of transferring from a bed to a chair and back, from a bed to a wheelchair and back, and coming to a standing position, if walking was the typical mode of locomotion for the patient. The chair/bed transfer component of the KMBI also assessed similar activities, but did not show a significant change after the intervention. This may be because the FIM scale has a more subdivided 7-graded consecutive scoring system than K-MBI, which has 5-graded stepwise scoring system.

The MUS scoring system examines 14 anatomical sites and assesses morphological change, inflammation, and effusion, consequently scoring 61 items. This system displayed substantial reliability and reproducibility [17]. Since assessing 61 items is time consuming and unnecessary, we selected 8 relevant items that had a probability of change. While the other items showed no change after treatment, Doppler activity was increased in the medial joint where ESWT was applied. The increase in Doppler activity was site-specific, but was observed in various structures.

To date, several studies have reported angiogenesis associated with ESWT application for knee OA in animal models, but not in human studies. Therefore, this study is meaningful in that it shows hemodynamic changes in the knee joint in humans as a result of ESWT. This increased vascularization after ESWT might indirectly suggest tissue regeneration or a chondroprotective effect.

Increased Doppler activity was not sustained at 1 week after treatment, but reduction of pain and improvement of function were confirmed in the same period of time. In our study, it was not possible to determine if there was any histological change after the decrease in Doppler activity.

Our study has several limitations. First, this study had a small sample size; however, we will conduct a future study with a larger groups of subjects based on the results of this pilot study. Second, we evaluated only the shortterm outcomes. Wang et al. [11] reported that ESWT showed a time-dependent chondroprotective effect on knee OA in rats, with the most beneficial effect at 4 weeks after treatment. Thus, investigation of the long-term ef- fects on cartilage is needed in future studies. Additionally, we applied only 1 treatment protocol in the experimental group. There has been only 1 study on the ESWT protocol for human knee OA [21]. As such, to establish the optimal regimen with regard to dose, frequency, and location, further investigation is required.

In conclusion, the present study suggests that ESWT may reduce pain and improve function in chronic stroke patients with knee OA and may increase site-specific Doppler activity following ESWT, even though the increase is not sustained and seems to decrease with time. For these reasons, we conclude that ESWT could be one of the treatment methods appropriate for human knee OA. Although the results of this pilot study are very encouraging, further study focusing on the treatment regimen and morphological or hemodynamic cartilaginous changes with a larger sample size will be necessary.

\section{CONFLICT OF INTEREST}

No potential conflict of interest relevant to this article was reported.

\section{REFERENCES}

1. Brundtland GH. Reducing risks to health, promoting healthy life. JAMA 2002;288:1974.

2. Kim I, Kim HA, Seo YI, Song YW, Jeong JY, Kim DH. The prevalence of knee osteoarthritis in elderly community residents in Korea. J Korean Med Sci 2010; 25:293-8.

3. Doruk P. The impact of knee osteoarthritis on rehabilitation outcomes in hemiparetic stroke patients. J Back Musculoskelet Rehabil 2013;26:207-11.

4. Zhang W, Moskowitz RW, Nuki G, Abramson S, Altman RD, Arden N, et al. OARSI recommendations for the management of hip and knee osteoarthritis. Part II: OARSI evidence-based, expert consensus guidelines. Osteoarthr Cartil 2008;16:137-62.

5. Wang CJ. An overview of shock wave therapy in musculoskeletal disorders. Chang Gung Med J 2003; 26:220-32.

6. Frisbie DD, Kawcak CE, McIlwraith CW. Evaluation of the effect of extracorporeal shock wave treatment on experimentally induced osteoarthritis in middle carpal joints of horses. Am J Vet Res 2009;70:449-54. 
7. Revenaugh MS. Extracorporeal shock wave therapy for treatment of osteoarthritis in the horse: clinical applications. Vet Clin North Am Equine Pract 2005;21:60925.

8. Mueller M, Bockstahler B, Skalicky M, Mlacnik E, Lorinson D. Effects of radial shockwave therapy on the limb function of dogs with hip osteoarthritis. Vet Rec 2007;160:762-5.

9. Dahlberg J, Fitch G, Evans RB, McClure SR, Conzemius $M$. The evaluation of extracorporeal shockwave therapy in naturally occurring osteoarthritis of the stifle joint in dogs. Vet Comp Orthop Traumatol 2005;18:147-52.

10. Wang CJ, Sun YC, Siu KK, Wu CT. Extracorporeal shockwave therapy shows site-specific effects in osteoarthritis of the knee in rats. J Surg Res 2013;183:612-9.

11. Wang CJ, Sun YC, Wong T, Hsu SL, Chou WY, Chang HW. Extracorporeal shockwave therapy shows timedependent chondroprotective effects in osteoarthritis of the knee in rats. J Surg Res 2012;178:196-205.

12. Zhao Z, Ji H, Jing R, Liu C, Wang M, Zhai L, et al. Extracorporeal shock-wave therapy reduces progression of knee osteoarthritis in rabbits by reducing nitric oxide level and chondrocyte apoptosis. Arch Orthop Trauma Surg 2012;132:1547-53.

13. Carlsson AM. Assessment of chronic pain. I: Aspects of the reliability and validity of the visual analogue scale. Pain 1983;16:87-101.

14. Loyola-Sanchez A, Richardson J, Beattie KA, OteroFuentes C, Adachi JD, MacIntyre NJ. Effect of lowintensity pulsed ultrasound on the cartilage repair in people with mild to moderate knee osteoarthritis: a double-blinded, randomized, placebo-controlled pilot study. Arch Phys Med Rehabil 2012;93:35-42.

15. Jung HY, Park BK, Shin HS, Kang YK, Pyun SB, Paik NJ, et al. Development of the Korean Version of Modified Barthel Index (K-MBI): multi-center study for subjects with stroke. J Korean Acad Rehabil Med 2007;31:28397.

16. Hamilton BB, Laughlin JA, Fiedler RC, Granger CV. Interrater reliability of the 7-level functional independence measure (FIM). Scand J Rehabil Med 1994;26:115-9.

17. Riecke BF, Christensen R, Torp-Pedersen S, Boesen M, Gudbergsen H, Bliddal H. An ultrasound score for knee osteoarthritis: a cross-sectional validation study. Osteoarthr Cartil 2014;22:1675-91.

18. Wang CJ. Extracorporeal shockwave therapy in musculoskeletal disorders. J Orthop Surg Res 2012;7:11.

19. Wang CJ, Hsu SL, Weng LH, Sun YC, Wang FS. Extracorporeal shockwave therapy shows a number of treatment related chondroprotective effect in osteoarthritis of the knee in rats. BMC Musculoskelet Disord 2013;14:44.

20. Zhao Z, Jing R, Shi Z, Zhao B, Ai Q, Xing G. Efficacy of extracorporeal shockwave therapy for knee osteoarthritis: a randomized controlled trial. J Surg Res 2013; 185:661-6.

21. Kim JH, Kim JY, Choi CM, Lee JK, Kee HS, Jung KI, et al. The dose-related effects of extracorporeal shock wave therapy for knee osteoarthritis. Ann Rehabil Med 2015;39:616-23.

22. Mayer-Wagner S, Ernst J, Maier M, Chiquet M, Joos H, Muller PE, et al. The effect of high-energy extracorporeal shock waves on hyaline cartilage of adult rats in vivo. J Orthop Res 2010;28:1050-6.

23. Dorotka R, Kubista B, Schatz KD, Trieb K. Effects of extracorporeal shock waves on human articular chondrocytes and ovine bone marrow stromal cells in vitro. Arch Orthop Trauma Surg 2003;123:345-8. 